\title{
Spatial characteristics of borromean, tango, samba and all-bound halo nuclei
}

\author{
M. T. Yamashita* ${ }^{*}$ T. Frederico ${ }^{\dagger}$ and Lauro Tomio** \\ ${ }^{*}$ Unidade Diferenciada de Itapeva, Universidade Estadual Paulista, 18409-010 Itapeva, Brazil. \\ †Departamento de Física, Instituto Tecnológico de Aeronáutica, Centro Técnico Aeroespacial, \\ 12228-900 São José dos Campos, Brazil. \\ **Instituto de Física Teórica, Universidade Estadual Paulista, 01405-900 São Paulo, Brazil.
}

\begin{abstract}
We report a renormalized zero-range interaction approach to estimate the size of generic weakly bound three-body systems where two particles are identical. We present results for the neutron-neutron root-mean-square distances of the halo nuclei ${ }^{6} \mathrm{He},{ }^{11} \mathrm{Li},{ }^{14} \mathrm{Be}$ and ${ }^{20} \mathrm{C}$, where the systems are taken as two halo neutrons with an inert point-like core. We also report an approach to obtain the neutron-neutron correlation function in halo nuclei. In this case, our results suggest a review of the corresponding experimental data analysis.
\end{abstract}

Keywords: few-body, root-mean-square radii, halo nuclei, correlation

PACS: 21.45.+v, 27.20.+n, 25.75.Gz

The quantum description of weakly bound three-body systems is universal and can be defined by few low-energy physical scales [1]. Using this concept, we consider a renormalized three-body formalism to estimate the size of generic weakly bound threebody systems $A A B$, where the two particles $A$ are identical. Next, the approach is applied to light exotic nuclei modeled as two neutrons and a core $(n-n-\mathscr{A})$. Finally, we will report some of our results for the mean-square radii of the two halo neutrons and also for the corresponding correlation function.

The results are derived from a low-energy universal scaling function that depends on the mass ratio of the neutron and the core, as well as on the nature of the subsystems, bound or virtual. The model consider a minimal number of physical inputs, which are directly related to observables: the two-neutron separation energy $S(2 n)=-E 3$, the neutron-neutron and neutron-core $s$-wave scattering lengths (or the corresponding virtual or bound energies).

The three-body system description is made by using Jacobi coordinates, where $\vec{q}_{i}$ is the relative momentum of the particle $i$ to the center-of-mass (CM) of the pair $j k$ and $\vec{p}_{i}$ is the relative momentum of the pair $j k . \vec{R}_{i}$ and $\vec{r}_{i}$ are, respectively, the positions canonically conjugated to the momenta. In the equations we consider $j \equiv k \equiv A$ and $i \equiv B$. We will show in a detailed form the formalism used to calculate the root-meansquare (rms) radii between the particles $j$ and $k$, using the corresponding form factor obtained from the Fourier transform of the density as a function of the relative distance. Similarly, one can obtain the rms distances between the other pair of particles.

The rms radii of the particles $j$ and $k$ are given by

$$
\left\langle r_{A A}^{2}\right\rangle=-\left.6 \frac{d F_{A A}\left(Q^{2}\right)}{d Q^{2}}\right|_{Q^{2}=0},
$$

CP884, VI Latin American Symposium on Nuclear Physics and Applications,

edited by O. Civitarese, C. Dorso, G. García Bermúdez, A. J. Kreiner, A. J. Pacheco, and N. N. Scoccola (C) 2007 American Institute of Physics 978-0-7354-0388-8/07/\$23.00 
where $F_{A A}\left(Q^{2}\right)$ is the form factor that, in terms of the wave functions $\Psi$ of the system in the momentum space, is given by (note that the particle $B$ is the spectator one)

$$
F_{A A}\left(Q^{2}\right)=\int d^{3} q_{i} d^{3} p_{i}\left\langle\vec{q}_{i}, \vec{p}_{i}+\frac{\vec{Q}}{2} \mid \Psi\right\rangle\left\langle\vec{q}_{i}, \vec{p}_{i}-\frac{\vec{Q}}{2} \mid \Psi\right\rangle .
$$

The Schrödinger equation of a three-body system with separable two-body potentials $v_{\alpha}=\lambda_{\alpha}\left|\chi_{\alpha}\right\rangle\left\langle\chi_{\alpha}\right|[\alpha \equiv(j k),(k i),(i j)]$, with $H_{0}$ the free-hamiltonian, is

$$
\begin{aligned}
& \left(H_{0}+\sum_{\alpha=i, j, k} \lambda_{\alpha}\left|\chi_{\alpha}\right\rangle\left\langle\chi_{\alpha}\right|\right)|\Psi\rangle=E|\Psi\rangle \\
& \left(E-H_{0}\right)|\Psi\rangle=\sum_{\alpha=i, j, k} \lambda_{\alpha}\left|\chi_{\alpha}\right\rangle\left\langle\chi_{\alpha} \mid \Psi\right\rangle .
\end{aligned}
$$

Using the Dirac- $\delta$ interaction $\left\langle\vec{p}_{\alpha} \mid \chi\right\rangle=1$, we can write the wave function in the base $\left|\vec{q}_{i}, \vec{p}_{i}\right\rangle$ in terms of the spectator functions $f_{\alpha}\left(\vec{q}_{\alpha}\right)=\lambda_{\alpha}\left\langle\vec{q}_{\alpha} \chi_{\alpha} \mid \Psi\right\rangle$ :

$$
\left\langle\vec{q}_{i}, \vec{p}_{i} \mid \Psi\right\rangle=\frac{f_{i}\left(\left|\vec{q}_{i}\right|\right)+f_{j}\left(\left|\vec{p}_{i}-\frac{\vec{q}_{i}}{2}\right|\right)+f_{k}\left(\left|\vec{p}_{i}+\frac{\vec{q}_{i}}{2}\right|\right)}{\left|E_{3}\right|+H_{0}},
$$

where $f_{i}, f_{j}$ e $f_{k}$ are, respectively, the spectator functions for the particles $i, j$ and $k$; and $E_{3}$ is the three-body binding energy. From (5) and (2) we obtain $F_{A A}\left(Q^{2}\right)$ :

$$
\begin{aligned}
F_{A A}\left(Q^{2}\right) & =\int d^{3} q_{i} d^{3} p_{i}\left(f_{i}\left(\left|\vec{q}_{i}\right|\right)+f_{j}\left(\left|\vec{p}_{i}+\frac{\vec{Q}}{2}-\frac{\vec{q}_{i}}{2}\right|\right)+f_{k}\left(\left|\vec{p}_{i}+\frac{\vec{Q}}{2}+\frac{\vec{q}_{i}}{2}\right|\right)\right) \\
& \times \frac{\left(f_{i}\left(\left|\vec{q}_{i}\right|\right)+f_{j}\left(\left|\vec{p}_{i}-\frac{\vec{Q}}{2}-\frac{\vec{q}_{i}}{2}\right|\right)+f_{k}\left(\left|\vec{p}_{i}-\frac{\vec{Q}}{2}+\frac{\vec{q}_{i}}{2}\right|\right)\right)}{\left(\left|E_{3}\right|+H_{0}\right)\left(\left|E_{3}\right|+H_{0}^{\prime}\right)},
\end{aligned}
$$

where

$$
H_{0} \equiv \frac{\left|\vec{p}_{i}+\frac{\vec{Q}}{2}\right|^{2}}{2 m_{A A}}+\frac{q_{i}^{2}}{2 m_{A A, B}}, \quad H_{0}^{\prime} \equiv \frac{\left|\vec{p}_{i}-\frac{\vec{Q}}{2}\right|^{2}}{2 m_{A A}}+\frac{q_{i}^{2}}{2 m_{A A, B}},
$$

with $m_{A A}=m_{A} / 2$ and $m_{A A, B}=2 m_{B} m_{A} /\left(2 m_{A}+m_{B}\right)$.

In an analogous way we can write the eqs. (1) and (2) for $\left\langle r_{A B}^{2}\right\rangle$ :

$$
\begin{gathered}
\left\langle r_{A B}^{2}\right\rangle=-\left.6 \frac{d F_{A B}\left(Q^{2}\right)}{d Q^{2}}\right|_{Q^{2}=0} . \\
F_{A B}\left(Q^{2}\right)=\int d^{3} q_{k} d^{3} p_{k}\left\langle\vec{q}_{k}, \vec{p}_{k}+\frac{\vec{Q}_{2}}{2} \mid \Psi\right\rangle\left\langle\vec{q}_{k}, \vec{p}_{k}-\frac{\vec{Q}}{2} \mid \Psi\right\rangle .
\end{gathered}
$$

Remembering that $i, j$ and $k$ correspond, respectively, to the spectator particles $B, A$, and $A$, the wave function in the base $\left|\vec{q}_{k}, \vec{p}_{k}\right\rangle$ is given by the following equation: 


$$
\left\langle\vec{q}_{k}, \vec{p}_{k} \mid \Psi\right\rangle=\frac{f_{i}\left(\left|\vec{p}_{k}-\frac{\mathscr{A}}{\mathscr{A}+1} \vec{q}_{k}\right|\right)+f_{j}\left(\left|\vec{p}_{k}+\frac{1}{\mathscr{A}+1} \vec{q}_{k}\right|\right)+f_{k}\left(\left|\vec{q}_{k}\right|\right)}{\left|E_{3}\right|+p_{k}^{2} \frac{\mathscr{A}+1}{2 \mathscr{A}}+q_{k}^{2} \frac{\mathscr{A}+2}{2(\mathscr{A}+1)}} .
$$

where $\mathscr{A}=m_{B} / m_{A}$ is the mass ratio of the particles $A$ and $B$

The rms distances of $A$ and $B$ to the three-body CM are calculated using the respective form factors and are given by

$$
\begin{aligned}
& \left\langle r_{A}^{2}\right\rangle \equiv\left(\frac{m_{A}+m_{B}}{2 m_{A}+m_{B}}\right)^{2}\left\langle R_{k}^{2}\right\rangle=-\left.6\left(\frac{m_{A}+m_{B}}{2 m_{A}+m_{B}}\right)^{2} \frac{d F_{A}\left(Q^{2}\right)}{d Q^{2}}\right|_{Q^{2}=0}, \\
& F_{A}\left(Q^{2}\right)=\int d^{3} q d^{3} p\left\langle\vec{q}_{k}+\frac{\vec{Q}}{2}, \vec{p}_{k} \mid \Psi\right\rangle\left\langle\vec{q}_{k}-\frac{\vec{Q}}{2}, \vec{p}_{k} \mid \Psi\right\rangle
\end{aligned}
$$

and

$$
\begin{aligned}
& \left\langle r_{B}^{2}\right\rangle \equiv\left(\frac{2 m_{A}}{2 m_{A}+m_{B}}\right)^{2}\left\langle R_{i}^{2}\right\rangle=-\left.6\left(\frac{2 m_{A}}{2 m_{A}+m_{B}}\right)^{2} \frac{d F_{B}\left(Q^{2}\right)}{d Q^{2}}\right|_{Q^{2}=0}, \\
& F_{B}\left(Q^{2}\right)=\int d^{3} q_{i} d^{3} p_{i}\left\langle\vec{q}_{i}+\frac{\vec{Q}}{2}, \vec{p}_{i} \mid \Psi\right\rangle\left\langle\vec{q}_{i}-\frac{\vec{Q}}{2}, \vec{p}_{i} \mid \Psi\right\rangle .
\end{aligned}
$$

Next, we consider units such that $\hbar=1$ and $m_{A}=1$. The spectator function are given in dimensionless units, using a subtraction approach required to regularize the equations, as follows:

$$
\begin{aligned}
f_{j}(\vec{q}) & =\left(\frac{\mathscr{A}+1}{2 \mathscr{A}}\right)^{3 / 2} \frac{1}{\pi}\left(\sqrt{|\varepsilon|+\frac{q^{2}(\mathscr{A}+2)}{2(\mathscr{A}+1)}} \mp \sqrt{\varepsilon_{A B}}\right)^{-1} \int_{0}^{\infty} k^{2} d k \int_{-1}^{1} d y \\
& \times\left[\frac{1}{|\varepsilon|+q^{2}+\frac{\mathscr{A}+1}{2 \mathscr{A}} k^{2}+k q y}-\frac{1}{1+q^{2}+\frac{\mathscr{A}+1}{2 \mathscr{A}} k^{2}+k q y}\right] f_{i}(\vec{k}) \\
& -\int_{-1}^{1}\left[\frac{1}{|\varepsilon|+\frac{(\mathscr{A}+1)}{2 \mathscr{A}} q^{2}+\frac{(\mathscr{A}+1)}{2 \mathscr{A}} k^{2}+\frac{1}{\mathscr{A}} k q y}-\frac{1}{1+\frac{\mathscr{A}+1)}{2 \mathscr{A}} q^{2}+\frac{(\mathscr{A}+1)}{2 \mathscr{A}} k^{2}+\frac{1}{\mathscr{A}} k q y}\right] f_{j}(\vec{k}) . \\
f_{i}(\vec{q}) & =\frac{2}{\pi}\left(\sqrt{|\varepsilon|+\frac{(\mathscr{A}+2)}{2 \mathscr{A}} q^{2}} \mp \sqrt{\varepsilon_{A A}}\right)^{-1} \int_{0}^{\infty} k^{2} d k \\
& \times \int_{-1}^{1} d y\left[\frac{1}{|\varepsilon|+\frac{(\mathscr{A}+1)}{2 \mathscr{A}} q^{2}+k^{2}+k q y}-\frac{1}{1+\frac{(\mathscr{A}+1)}{2 \mathscr{A}} q^{2}+k^{2}+k q y}\right] f_{j}(\vec{k}) .
\end{aligned}
$$


In the above, we first take $\mu_{(3)}^{2}$ as our energy-subtraction point required to regularize the equations [2]); next, we consider all the energies $\varepsilon$ and momenta $q, k$ in units of $\mu_{(3)}^{2}$ and $\mu_{(3)}$, respectively. Note that the spectator functions $f_{j}$ and $f_{k}$ are equal. In front of the energy square-root, the - sign refers to a bound state and the + to a virtual one.

According to the two-body interactions we can have four types of a $A A B$ system: borromean configuration [3], when all the two-body subsystems are unbound; tango configuration [4], when we have two unbound and one bound two-body subsystems; samba configuration [5], when just one two-body subsystems is unbound; and all-bound configuration, when there is no unbound subsystems.

A qualitative analysis of eqs. (13) and (14) shows what happens with the sizes of the three-body systems when we change the two-body interactions. In the all-bound configuration the signs in front of $\sqrt{\varepsilon_{A B}}$ and $\sqrt{\varepsilon_{A A}}$ are both negative, the samba type has a negative sign in front of $\sqrt{\varepsilon_{A B}}$ and a positive in front of $\sqrt{\varepsilon_{A A}}$, the tango type has a negative sign in front of $\sqrt{\varepsilon_{A A}}$ and a positive in front of $\sqrt{\varepsilon_{A B}}$; finally, for the borromean configuration the signs in front of $\sqrt{\varepsilon_{A B}}$ and $\sqrt{\varepsilon_{A A}}$ are both positive. Then, the sequence according to the kernel attraction is: all-bound $>$ samba $>$ tango $>$ borromean. These differences are reflected in the sizes of the system as follows (for a same three-body energy): in the case of a more attractive kernel the particles can be more separated and produce a three-body bound state, for a less attractive kernel the particles should stay closer. In this way, for a same three-body energy the sizes of the systems vary as: allbound $>$ samba $>$ tango $>$ borromean.

For a three-body system with binding energy $E_{3}$, in the scaling limit [1], one general three-body physical observable $\mathscr{O}$, with dimension of energy to the power $\eta$, at a particular energy $E$, can be written as a function $\mathscr{F}$ of the ratios between the two and three-body energies, such that

$$
\mathscr{O}\left(E, E_{3}, E_{A A}, E_{A B},\right)=\left(E_{3}\right)^{\eta} \mathscr{F}\left(\sqrt{\frac{E}{E_{3}}}, \pm \sqrt{\frac{E_{A A}}{E_{3}}}, \pm \sqrt{\frac{E_{A B}}{E_{3}}}, \mathscr{A}\right)
$$

The two-body energies $E_{A \gamma}(\gamma=A, B)$, are negative quantities, corresponding to bound or virtual states. The nature of such two-body state, bound or virtual, is revealed in the momentum space, such that we have a bound state when $\sqrt{\left|E_{A B}\right|}$ is positive and a virtual state when $\sqrt{\left|E_{A B}\right|}$ is negative. So, in equation (15), the signs + or - mean a bound or virtual two-body subsystem, respectively. The different radii of the bound $A A B$ system are functions defined from the eq. (15) with $E=E_{3}$, which depend on the mass ratio, $\mathscr{A}$, the ratios of the two and three-body energies and the kind of subsystem interactions (bound or virtual).

The above approach for a generic three-body system can be applied for the case of halo nuclei, where we have a pointlike inert core $(\mathscr{A})$ and two weakly bound neutrons (n). In this case, we shown in Table 1 the results of our calculations of the root-meansquare distances for the two neutrons of the core, for the halo-nuclei ${ }^{6} \mathrm{He},{ }^{11} \mathrm{Li},{ }^{14} \mathrm{Be}$ and ${ }^{20} \mathrm{C}$ (for an extended version of this table, see Ref. [5]).

Experimentally, the size of a halo nucleus can be measured using a neutron-neutron correlation function, $C_{n n}$. For the $n n C$ three-body system, $C_{n n}$ is given by [6] 
TABLE 1. Neutron-neutron root-mean-square distances in halo nuclei. The cores are given in the first column, the absolute values of the three-body ground state energies $E_{3}$ are given in the second column. $-E_{3}$ is equal to the two-neutron separation energy $S(2 n)$. In the third column we give our input values for $-E_{n \mathscr{A}}$. For bound two-body subsystem $n \mathscr{A}$, we have $-E_{n \mathscr{A}}$ equal to the one-neutron separation energy $S(n)$. The virtual states are indicated by (v), and the $n n$ virtual state energy is taken as $-143 \mathrm{keV}$. For the experimental values, in the last column, see Ref. [5].

\begin{tabular}{lllcc}
\hline Core $(\mathscr{A})$ & $-E_{3}(\mathrm{MeV})$ & $-E_{n \mathscr{A}}(\mathrm{MeV})$ & $\sqrt{\left\langle r_{n n}^{2}\right\rangle}(\mathrm{fm})$ & $\sqrt{\left\langle r_{n n}^{2}\right\rangle}$ exp $(\mathrm{fm})$ \\
\hline${ }^{4} \mathrm{He}$ & 0.973 & 0 & 5.1 & $5.9 \pm 1.2$ \\
${ }^{9} \mathrm{Li}$ & 0.29 & $0.05(\mathrm{v})$ & 8.5 & $6.6 \pm 1.5$ \\
${ }^{12} \mathrm{Be}$ & 1.337 & 0 & 4.6 & $5.4 \pm 1.0$ \\
${ }^{18} \mathrm{C}$ & 3.50 & 0.53 & 3.0 & - \\
\hline
\end{tabular}

$$
\begin{gathered}
C_{n n} \equiv C\left(\vec{p}_{\mathscr{A}}\right)=\frac{\int d^{3} q_{\mathscr{A}}\left|\Phi\left(\vec{q}_{\mathscr{A}}, \vec{p}_{\mathscr{A}}\right)\right|^{2}}{\int d^{3} q_{\mathscr{A}} \rho\left(\vec{q}_{n^{\prime}}\right) \rho\left(\vec{q}_{n)}\right.}, \\
\vec{q}_{n^{\prime}} \equiv \vec{p}_{\mathscr{A}}-\frac{\vec{q}_{\mathscr{A}}}{2} \text { and } \vec{q}_{n} \equiv-\vec{p}_{\mathscr{A}}-\frac{\vec{q}_{\mathscr{A}}}{2}
\end{gathered}
$$

where the one-body density is

$$
\rho\left(\vec{q}_{n^{\prime}}\right)=\int d^{3} q_{n}\left|\Phi\left(-\vec{q}_{n^{\prime}}-\vec{q}_{n}, \frac{\vec{q}_{n^{\prime}}-\vec{q}_{n}}{2}\right)\right|^{2} .
$$

$\Phi \equiv \Phi\left(\vec{q}_{\mathscr{A}}, \vec{p}_{\mathscr{A}}\right)$ is the corresponding breakup amplitude of three-body wave function including the FSI between the neutrons. $\vec{q}_{\mathscr{A}}$ is the relative momentum between the core $\mathscr{A}$ and the CM of the $n n$ subsystem; and $\vec{p}_{\mathscr{A}}$ the relative momentum between the neutrons.

The FSI is introduced directly in the inner product $\Phi \equiv\left\langle\vec{q}_{\mathscr{A}} ; \vec{p}_{\mathscr{A}}{ }^{(-)} \mid \Psi\right\rangle$, where the ket $\left|\vec{p}_{\mathscr{A}}{ }^{(-)}\right\rangle$refers to the $n n$ scattered wave given by the Lippmann-Schwinger equation. The correlation function, calculated with the distorted-wave amplitude, assumes a sudden breakup of the halo as the main reaction mechanism. The halo is considered as a coherent source of neutrons. In our picture, the slow halo motion decouples from the fast motion of the core in the field of the target. The distorted wave amplitude $\Phi$ is given by

$$
\Phi=\Psi\left(\vec{q}_{\mathscr{A}}, \vec{p}_{\mathscr{A}}\right)+\frac{1 /\left(2 \pi^{2}\right)}{\sqrt{E_{n n}}-i p_{\mathscr{A}}} \int d^{3} p \frac{\Psi\left(\vec{q}_{\mathscr{A}}, \vec{p}\right)}{p_{\mathscr{A}}^{2}-p^{2}+i \varepsilon},
$$

where $\Psi$ is the three-body wave function [5]. $E_{n n}$ is the $n n$ virtual state energy.

The interference effect produced by the inclusion of FSI originates a minimum for $C_{n n}$, pushing the asymptotic limit to much larger values of $p_{\mathscr{A}}$ than the ones considered in the asymptotic normalization of the experimental data (see Ref. [6]). 
In summary, we report here a formalism and results obtained for the sizes of generic three-body systems. If we consider the same three-body energy for all the configurations, the following sequence is applied for the mean-square radii $(\mathrm{msr})$ : $\mathrm{msr}_{\text {borromean }}<\mathrm{msr}_{\text {tango }}<\mathrm{msr}_{\text {samba }}<\mathrm{msr}_{\text {all-bound }}$. With the available low-energy twobody observables, in our scaling limit formalism, we have estimated the mean-square distances for the nuclei ${ }^{6} \mathrm{He},{ }^{11} \mathrm{Li},{ }^{14} \mathrm{Be}$ and ${ }^{20} \mathrm{C}$, in a model where they are described as inert cores with two halo neutrons. Finally, we presented our model approach for the two-neutron correlation function $C_{n n}$. As detailed in ref. [6], our results for $C_{n n}$ calls for a different asymptotic normalization of the experimental data. We believe that our qualitative picture, evidencing a minimum of $C_{n n}$, will survive in a more realistic three-body approach.

We thank partial support received from FAPESP and CNPq. MTY thanks support from the organizers of SLAFNAP6.

\section{REFERENCES}

1. M. T. Yamashita, T. Frederico, A. Delfino and L. Tomio, Phys. Rev. A66, 052702 (2002).

2. S. K. Adhikari, T. Frederico and I. D. Goldman, Phys. Rev. Lett. 74, 487 (1995);

S. K. Adhikari and T. Frederico, Phys. Rev. Lett. 74, 4572 (1995).

3. A. S. Jensen, K. Riisager, D. V. Fedorov, E. Garrido, Rev. Mod. Phys. 76, 215 (2004).

4. F. Robichaux, Phys. Rev. A60, 1706 (1999).

5. M. T. Yamashita, L. Tomio and T. Frederico, Nucl. Phys. A735, 40 (2004) and references therein.

6. M. T. Yamashita, T. Frederico and Lauro Tomio, Phys. Rev. C72, 011601 (2005) and references therein. 
Copyright of AIP Conference Proceedings is the property of American Institute of Physics and its content may not be copied or emailed to multiple sites or posted to a listserv without the copyright holder's express written permission. However, users may print, download, or email articles for individual use. 